Case Reports
in Dermatology
Case Rep Dermatol 2021;13:248-256

DOI: $10.1159 / 000515246$

Published online: May 4, 2021
(C) 2021 The Author(s)

Published by S. Karger AG, Basel www.karger.com/cde

This article is licensed under the Creative Commons Attribution-NonCommercial 4.0 International License (CC BY-NC) (http://www.karger.com/Services/OpenAccessLicense). Usage and distribution for commercial purposes requires written permission.

\title{
Atopic Dermatitis Treated Safely with Dupilumab during Pregnancy: A Case Report and Review of the Literature
}

\author{
Yolanka Lobo ${ }^{a, b}$ Ruby C. Lee ${ }^{a, b}$ Lynda Spelman ${ }^{a, b}$ \\ aVeracity Clinical Research, Brisbane, QLD, Australia; ${ }^{b}$ Gabba Dermatology, \\ Brisbane, QLD, Australia
}

\section{Keywords}

Atopic dermatitis · Dupilumab · Pregnancy

\begin{abstract}
Dupilumab is currently the only biologic treatment approved for moderate-to-severe atopic dermatitis. Though limited, available clinical data describing dupilumab use in pregnancy have not identified a drug-associated risk of major birth defects, miscarriage, or adverse maternal or fetal outcomes. Systemic treatment in pregnant women with atopic dermatitis is currently restricted to corticosteroids, cyclosporine $\mathrm{A}$, and azathioprine. Atopic dermatitis often has a deleterious course in pregnancy which can cause substantial distress and significantly impact on global health and quality of life. We report a case of severe atopic dermatitis treated safely with dupilumab during pregnancy with no adverse maternal or fetal outcomes observed. Our case highlights that dupilumab use in pregnancy has its place but should always be preceded by careful assessment of the risks and benefits. Clinicians are encouraged to enroll their patients in relevant pregnancy registry studies to monitor outcomes in women exposed to dupilumab during pregnancy and lactation.




\section{Case Reports in Dermatology}

Case Rep Dermatol 2021;13:248-256

DOI: $10.1159 / 000515246$

(c) 2021 The Author(s). Published by S. Karger AG, Basel www.karger.com/cde

Lobo et al.: Atopic Dermatitis Treated Safely with Dupilumab during Pregnancy: A Case Report and Review of the Literature

\section{Introduction}

Dupilumab is a fully human monoclonal antibody of the immunoglobulin G (IgG) 4 subclass that inhibits interleukin (IL)-4 and IL-13 signalling by binding to the shared IL-4R $\alpha$ subunit of the IL-4 and IL-13 receptor complexes. Thus, dupilumab inhibits the action of two key cytokines involved in the pathogenesis of atopic dermatitis (AD). It is currently the only biologic treatment approved for patients with moderate-to-severe AD who are candidates for systemic therapy. In pregnancy, AD often has a deleterious course which can cause substantial distress and significantly impact on global health and quality of life [1-3]. According to the position paper from the European Task Force on Atopic Dermatitis (ETFAD), systemic treatment in pregnant and lactating women with $\mathrm{AD}$ should be restricted to corticosteroids, cyclosporine A, and, in selected cases, azathioprine [4]. Dupilumab, like other IgG antibodies, is expected to cross the placental barrier and be excreted in human breast milk [5]. Though limited, available clinical data describing dupilumab use in pregnancy have not identified a drug-associated risk of major birth defects, miscarriage, or adverse maternal or fetal outcomes. We report a case of severe AD treated safely with dupilumab during pregnancy. Additionally, we summarize the available literature describing dupilumab use in pregnancy.

\section{Case Report}

A 36-year-old gravida 1 para 1 woman presented to our dermatology clinic with severe AD since early childhood. Her past medical history was significant for allergic rhinoconjunctivitis and asthma. Previous treatments for AD included high-potency topical corticosteroids (TCS), systemic corticosteroids (SCS), narrowband ultraviolet-B (UVB) phototherapy, methotrexate, and a 12-month course of cyclosporine A. On examination, she had diffuse AD affecting $86 \%$ of her total body surface area, with an Eczema Area and Severity Index (EASI) score of 67.4, and an Investigator Global Assessment score of 4, indicating severe disease. The patient was commenced on dupilumab with a loading dose of $600 \mathrm{mg}$ followed by $300 \mathrm{mg}$ every other week. Within four weeks of treatment, her AD had improved significantly. At week 16 of treatment, she had an EASI score of 1.7. Due to eye-related adverse effects, dosing was decreased to 3-weekly with eventual resolution of symptoms. She became pregnant 12 months later but continued to receive dupilumab until 24 weeks and 4 days gestation after careful risk-benefit assessment. Apart from gestational diabetes, there were no other antenatal complications. Within a few weeks of cessation, she experienced a severe flare of AD, reflected by an EASI score of 40.1, which was refractory to emollients, oral antibiotics and UVB phototherapy. She decided against treatment with TCS and SCS. At 37 weeks and 4 days gestation, a healthy female infant (weight 3,120 g, length $48.5 \mathrm{~cm}$ ) was born via an uncomplicated spontaneous vaginal delivery and shortly after, dupilumab was recommenced. The patient chose not to breastfeed while receiving dupilumab. Six weeks after reintroduction of dupilumab, she showed significant skin clearance with an EASI score of 4.2. The influence of dupilumab on the severity of AD before, during and after pregnancy, as reflected by the EASI score, is shown in Fig. 1.

\section{Karger'=}




\section{Case Reports in Dermatology}

Case Rep Dermatol 2021;13:248-256

DOI: $10.1159 / 000515246$

(c) 2021 The Author(s). Published by S. Karger AG, Basel www.karger.com/cde

Lobo et al.: Atopic Dermatitis Treated Safely with Dupilumab during Pregnancy: A Case Report and Review of the Literature

\section{Discussion}

\section{Atopic Dermatitis in Pregnancy}

Atopic dermatitis accounts for $36-49 \%$ of all gestational dermatoses, making it the most common dermatosis encountered in pregnancy [6,7]. Pregnancy is often a trigger for AD and worsening of disease is typically reported during the second and third trimesters [4, 7]. In a normal pregnancy state, the balance between the T helper cell (Th) 1 and Th2 cytokine response is altered [8]. To prevent fetal rejection and thus reduce the risk of miscarriage, there is a shift from Th1 to Th2 response [9]. As the dominant response in $\mathrm{AD}$ is of the Th2 type, the immunological state of women during pregnancy is thought to worsen the severity of this disease [10].

There is no evidence that $\mathrm{AD}$ as a disease itself is a risk factor for adverse pregnancy outcomes but adequate control of disease during pregnancy may optimize maternal and fetal health. Management of pregnant or lactating women with $\mathrm{AD}$ is often challenging owing to the paucity of large clinical studies investigating possible effects of treatment on conception, pregnancy, the fetus, and lactation [4]. Physicians and patients tend to limit the use of topical and in particular systemic therapies during preconception, pregnancy, and lactation to avoid adverse drug reactions and presumed harm to the fetus. Results of a recent register-based study in Denmark showed that pregnant women with AD had an overall reduced usage of both topical and systemic medications when compared to the period prior to their pregnancy [11]. Additionally, increased use of prednisolone observed in this cohort may represent undertreatment of AD resulting in the need for systemic rescue therapy [11]. Untreated or inadequately treated AD can potentially place both mother and fetus at an increased risk of serious complications, such as eczema herpeticum, Staphylococcus aureus infections, and, albeit rare, neonatal septicemia $[11,12]$. Exacerbation of AD may also result in anxiety, mood changes, and impaired quality of life [4].

\section{Current Treatment Recommendations for Atopic Dermatitis in Pregnancy}

Topical corticosteroids, topical calcineurin inhibitors, and narrowband UVB phototherapy can be safely used during pregnancy for $\mathrm{AD}$ [4]. If unable to achieve sufficient control with these modalities, systemic therapy should be considered. Short courses of oral corticosteroids (up to $0.5 \mathrm{mg} / \mathrm{kg} / \mathrm{day}$ ) can be used as first-line when immediate or short-term control is needed for acute flare-ups or second-line when long-term therapy is required $[4,7]$. The ETFAD supports treatment with SCS for AD in pregnancy for a short duration $(<2-3$ weeks $)$ but only if adequately administered TCS and phototherapy have failed [4]. Cyclosporine A is considered a first-line systemic treatment option when topical treatments and phototherapy have failed and the need for better long-term disease control is indicated; however, it may increase the risk of low birth weight in newborns $[4,13]$. Whenever possible, the use of azathioprine in pregnancy should be avoided due to the availability of better alternatives. However, it may be continued in cases of severe AD when the patient is already receiving this treatment at the time of conception and when no other treatment options are available [4]. The dose of azathioprine should be reduced by 50 percent if it is continued during pregnancy [4].

\section{The Use of Biologic Therapies in Pregnancy}

Immunoglobulin $\mathrm{G}$ is the only major class of antibody that is transported across the placental barrier; IgG1 is the most transported antibody, and IgG4 is the second most transported antibody [14]. Fetal levels of IgG in umbilical venous blood are low in the first two trimesters

\section{Karger'=}




\section{Case Reports in Dermatology}

Case Rep Dermatol 2021;13:248-256

DOI: $10.1159 / 000515246$

(c) 2021 The Author(s). Published by S. Karger AG, Basel www.karger.com/cde

Lobo et al.: Atopic Dermatitis Treated Safely with Dupilumab during Pregnancy: A Case Report and Review of the Literature

of pregnancy and do not exceed maternal levels of IgG until the beginning of the third trimester [15]. At this time, active transport of IgG molecules across the placenta rapidly increases owing to the development of fetal Fc receptors [15, 16]. Given that placental transport of maternal antibodies during the first two trimesters of pregnancy is negligible, the use of tissue necrosis factor (TNF)- $\alpha$ inhibitors is generally considered safe during the first half of pregnancy without an increased risk of miscarriage or birth defects $[17,18]$. The Canadian Association of Gastroenterology recommends that anti-TNF therapy should be ceased at 2224 weeks of gestation in women with a low risk of inflammatory bowel disease (IBD) relapse and continued throughout pregnancy in all other women with IBD [19]. These recommendations are based on the assumption that the risks of anti-TNF therapy outweigh the risks of an IBD relapse during pregnancy with respect to maternal and fetal health [19].

There is a growing body of evidence which suggests that biologic agents can be used for the treatment of dermatological conditions such as psoriasis during pregnancy and lactation $[8,15]$. The majority of the data on biologic agents and pregnancy, including guidelines and recommendations, stem from gastroenterology and rheumatology literature due to the frequent use TNF- $\alpha$ inhibitors to treat IBD and rheumatoid arthritis [15]. Given that controlled clinical trials often exclude pregnant women from enrollment, data on newer biologic agents, such as ustekinumab (IL-12/23 inhibitor), secukinumab (IL-17 inhibitor), and ixekizumab (IL-17 inhibitor), are lacking. Evidence for the use of biologic therapies in pregnancy for dermatological conditions mostly originates from animal studies, case reports or case series, small retrospective studies, pregnancy registry studies, and surveillance programs [8]. Registries however can be subject to bias because adverse outcomes are more likely to be reported $[8,15]$. Overall, the current evidence indicates that biologic therapies appear to be safe in the first two trimesters of pregnancy, especially if the potential benefits of treatment outweigh the potential risks [8].

\section{The Use of Dupilumab in Pregnancy}

Currently, there are no published studies evaluating pregnancy and fetal outcomes in female patients treated with dupilumab [5, 20, 21]. Dupilumab, being an IgG4 antibody, is expected to cross the placental barrier and be transmitted to the fetus [4]. The sponsor has proposed placement of dupilumab in Pregnancy Category B1 [5]. According to the Australian Therapeutic Goods Administration's categorization system for prescribing medicines in pregnancy, category B1 drugs are those which have been taken by only a limited number of pregnant women and women of childbearing age, without an increase in the frequency of malformation or other observed direct or indirect harmful effects on the human fetus [22]. Placement in this category is supported based on the absence of adverse effects with respect to reproductive toxicity observed in cynomolgus monkeys using surrogate anti-IL-4R $\alpha$ antibodies [23]. No treatment-related effects on embryofetal survival, malformations, growth, functional development, or immunology were observed in the offspring during a monitoring period from birth to six months of age [5]. In addition, studies conducted in male and female mice using surrogate anti-IL-4R $\alpha$ antibodies showed no effect on fertility [5].

Recently, two case reports have depicted good maternal and fetal outcomes in two pregnant patients treated with dupilumab for $\mathrm{AD}[10,24]$. The first to be reported was a 35-yearold female in Germany who became pregnant eight months into treatment with dupilumab [10]. Dupilumab was ceased in her second week of pregnancy and topical therapy was initiated thereafter for three months with minimal effect. The patient decided to reintroduce dupilumab on her own. After a risk-benefit assessment, continuation of dupilumab was agreed

\section{Karger'=}




\section{Case Reports in Dermatology}

Case Rep Dermatol 2021;13:248-256

DOI: $10.1159 / 000515246$

(c) 2021 The Author(s). Published by S. Karger AG, Basel www.karger.com/cde

Lobo et al.: Atopic Dermatitis Treated Safely with Dupilumab during Pregnancy: A Case Report and Review of the Literature

upon by her treating physicians. Her AD had improved significantly after eight weeks of treatment and remained stable throughout her pregnancy. There were no reported pregnancy-related complications and at week 40 , a healthy baby girl was delivered. The child was breastfed for the next four months without complications. The second case involved a 28-year-old pregnant patient with severe AD treated with dupilumab in the United States of America [24]. After no response to TCS, SCS, and phototherapy, the patient received her first loading dose of dupilumab at 24 weeks gestational age. Her skin dramatically improved during her pregnancy and she had an uncomplicated delivery at 37 weeks and 6 days gestation. Apart from gestational diabetes and eye irritation for 1-2 days after injections, there were no other drug-related adverse effects or complications after the introduction of dupilumab.

In the opinion of the ETFAD, neither experimental data nor theoretical considerations indicate the teratogenicity of dupilumab [4]. The authors recommend against the use of dupilumab in pregnant and lactating women [4]. The European Respiratory Society and the Thoracic Society of Australia and New Zealand Task Force on the management of reproduction and pregnancy in women with airways diseases classifies dupilumab as "Possibly Safe" in prepregnancy, pregnancy, labor, and breastfeeding states [25]. This classification was based on data provided by case reports and the Xolair Pregnancy Registry (EXPECT) study which suggest that outcomes of pregnancies exposed to omalizumab in the first trimester do not differ significantly from other pregnant women with moderate-to-severe asthma. The authors comment that since the transport of monoclonal antibodies across the placental barrier increases as pregnancy progresses, any potential fetal effects are likely to be greater during the last two trimesters of pregnancy [25].

The European Medicines Agency (EMA) reported data from one phase $2 \mathrm{~b}$ and three phase 3 studies assessing all investigational indications for dupilumab including over 2,500 patients with $\mathrm{AD}$ [26]. As of the data cut-off date (27 April 2016), there were a total of 23 pregnancies in patients exposed to dupilumab resulting in seven live births of eight healthy infants (including one set of twins), two elective abortions, and six spontaneous abortions (SABs), with five pregnancies ongoing at the time of the data cut and three pregnancies lost to follow-up. Of the six patients with $S A B$, two had one or more factors known to increase the risk of SAB, i.e., elevated parathyroid hormone, clotting disorders, and a history of infertility [26]. In the EMA "Committee for Medicinal Products for Human Use assessment report on extension of marketing authorization and indication variation" for dupilumab in asthma treatment published in 2019, a summary of pregnancy outcomes was reported from one phase $2 \mathrm{~b}$ and two phase 3 studies [27]. A total of 19 pregnancies in patients exposed to dupilumab were reported resulting in eight healthy infants (including one set of twins), one infant with Turner's syndrome associated with bicuspid aortic valve, three elective abortions, and five SABs, with three pregnancies ongoing at the time of the data cut. Of the five patients with $S A B$, three were deemed to be unrelated to dupilumab given the presence of at least one risk factor for SAB, i.e., clotting disorders, prior use of infertility treatments, advanced age, and twin pregnancy [27]. The EMA assessment reports for dupilumab indicate that the $\mathrm{SAB}$ rate registered during the dupilumab studies in AD and asthma did not exceed that of the general population [26, 27]. Overall, no drug-associated risk of major birth defects, miscarriage, or adverse maternal or fetal outcomes could be determined due to the small number of pregnancies analysed [26, 27].

\section{Dupilumab and Breastfeeding}

There is no available evidence on the effects of dupilumab on the breastfed infant and for this reason the ETFAD does not support its use in lactating women [4]. Human IgG is known

\section{Karger'=}




\section{Case Reports in Dermatology}

Case Rep Dermatol 2021;13:248-256

DOI: 10.1159/000515246

(c) 2021 The Author(s). Published by S. Karger AG, Basel www.karger.com/cde

Lobo et al.: Atopic Dermatitis Treated Safely with Dupilumab during Pregnancy: A Case Report and Review of the Literature

to be excreted in breastmilk [8]. As experience during pregnancy and lactation is limited, monoclonal antibodies should only be used if conventional therapies for AD have failed [25]. Due to their high molecular weight, the secretion of biologic agents in breast milk is miniscule except during the first three days, when there are wide gaps between the breast alveolar cells which enable immunoglobulins to pass through [8]. Thus, it is better to avoid breastfeeding during this period [28]. There are no safety concerns regarding mothers treated with TNF- $\alpha$ inhibitors who breastfeed their infants due to the miniscule amounts secreted in breastmilk, and protein degradation by the acid and proteolytic enzymes in the infant's gut, thereby preventing gastrointestinal absorption [15]. Use of biologic agents and/or breastfeeding should therefore be avoided in the first few days of life and in preterm infants who are likely to have an immature gastrointestinal tract [8]. Extrapolation of breastfeeding safety data on TNF- $\alpha$ inhibitors to newer biologic agents such as dupilumab must be done with caution and only if the benefits outweigh the risks $[8,15]$.

\section{Dupilumab and Neonatal Considerations}

The effects of dupilumab exposure in pregnancy on the neonatal immune system are unknown. Patients with AD who receive biologic therapy during pregnancy should be advised of the potential for an impaired immune response in their newborns [15]. A fatal case of disseminated Bacillus Calmette-Guérin (BCG) infection was reported in an infant exposed to infliximab during pregnancy following a live BCG vaccination [29]. Dupilumab has not been shown to have adverse effects in adult patients who receive inactive vaccines [30]. In fact, patients treated with dupilumab who receive inactive vaccines develop normal immune responses against these antigens [30]. Although no data are available on live vaccinations and dupilumab treatment, some experts predict that there will be no safety issues for the infant, given that interleukin inhibitors have less effect on systemic immunity [30]. As biologic agents are more actively transported across the placental barrier in the second and third trimesters of pregnancy, the administration of live vaccinations should be avoided in newborns with exposure to biologic agents in the later trimesters for at least six months after delivery, or when the serum levels of biologic medications are no longer detectable [8, 15, 31]. Inactive vaccinations on the other hand are permitted and should follow the standard national immunization schedule $[8,15]$.

\section{Conclusion}

There are limited available data on the use of dupilumab in pregnant women to inform any drug-associated risk. Until more experience is available, systemic treatment in pregnant women with $\mathrm{AD}$ is restricted to corticosteroids, cyclosporine $\mathrm{A}$, and azathioprine. To our knowledge, this is the first case report of a pregnant patient with AD treated with dupilumab in Australia. Maternal and fetal outcomes were excellent. Our case highlights that dupilumab use in pregnancy has its place but should be preceded by careful assessment of whether the potential benefits justify the potential risks to the fetus. Clinicians are encouraged to enroll their patients in manufacturer-sponsored pregnancy registry studies or surveillance programs to monitor outcomes in women exposed to dupilumab during pregnancy. As such, all pregnancy outcomes, adverse or otherwise, should be reported to increase our understanding of the effects of dupilumab use during pregnancy and lactation.

\section{Karger'=}




\section{Case Reports in Dermatology}

\section{Acknowledgements}

The authors wish to thank their patient for allowing them to share her story.

\section{Statement of Ethics}

Written informed consent was obtained from the patient for publication of this case report and accompanying images in accordance with the World Medical Association Declaration of Helsinki.

\section{Conflict of Interest Statement}

Dr Lobo has served as a sub-investigator for AbbVie, Amgen, Boehringer Ingelheim, Bristol Myers Squibb, Dermira, Eli Lilly, Galderma, Janssen, Leo Pharma, Mayne, Novartis, Pfizer, Sanofi, Sun Pharmaceuticals, and Union Chimique Belge. Dr Lee has served as a sub-investigator for AbbVie, Amgen, Boehringer Ingelheim, Bristol Myers Squibb, Dermira, Eli Lilly, Galderma, Janssen, Leo Pharma, Mayne, Novartis, Pfizer, Sanofi, Sun Pharmaceuticals, and Union Chimique Belge. Dr Spelman has served on advisory boards for AbbVie, Eli Lilly, Galderma, Janssen and Novartis; has served as an investigator for AbbVie, Amgen, Anacor, Ascend Biopharmaceuticals, Astellas, Australian Wool Innovation Limited, Blaze Bioscience, Boehringer Ingelheim, Bristol Myers Squibb, Botanix, Celgene, Dermira, Eli Lilly, Galderma, Genentech, GlaxoSmithKline, Janssen, Kythera, Leo Pharma, Mayne, Merck, Novartis, Pfizer, Phosphagenics, Regeneron, Sanofi, Sun Pharmaceuticals, Trius, and Union Chimique Belge; and has received sponsored travel from Abbott, Novartis, and Janssen.

\section{Funding Sources}

The authors received no financial support or sponsorship for the research and/or authorship of this case report.

\section{Author Contributions}

Yolanka Lobo: conception of the work; design of the work; acquisition and analysis of data; drafting the work; revising the work for important intellectual content; final approval of the version to be published; agreed to be accountable for all aspects of the work in ensuring that questions related to the accuracy or integrity of any part of the work are appropriately investigated and resolved. Ruby Lee: conception of the work; design of the work; acquisition and analysis of data; drafting the work; revising the work for important intellectual content; final approval of the version to be published; agreed to be accountable for all aspects of the work in ensuring that questions related to the accuracy or integrity of any part of the work are appropriately investigated and resolved. Lynda Spelman: conception of the work; design of the work; acquisition and analysis of data; drafting the work; revising the work for important intellectual content; final approval of the version to be published; agreed to be accountable for all aspects of the work in ensuring that questions related to the accuracy or integrity of any part of the work are appropriately investigated and resolved.

\section{Karger'=}




\section{Case Reports in Dermatology}

\begin{tabular}{l|l}
\hline DOI: $10.1159 / 000515246$ & $\begin{array}{l}\text { (c) } 2021 \text { The Author(s). Published by S. Karger AG, Basel } \\
\text { www.karger.com/cde }\end{array}$
\end{tabular}

Lobo et al.: Atopic Dermatitis Treated Safely with Dupilumab during Pregnancy: A Case Report and Review of the Literature

\section{References}

1 Kage P, Simon JC, Treudler R. Atopic dermatitis and psychosocial comorbidities. J Dtsch Dermatol Ges. 2020 Feb;18(2):93-102.

2 Treudler R. [Allergic diseases in pregnancy. Overview of diagnosis and therapy]. Hautarzt. 2010 Dec;61(12):1027-33.

3 Treudler R, Zeynalova S, Riedel-Heller SG, Zuelke AE, Roehr S, Hinz A, et al. Depression, anxiety and quality of life in subjects with atopic eczema in a population-based cross-sectional study in Germany. J Eur Acad Dermatol Venereol. 2020 Apr;34(4):810-6.

4 Vestergaard C, Wollenberg A, Barbarot S, Christen-Zaech S, Deleuran M, Spuls P, et al. European task force on atopic dermatitis position paper: treatment of parental atopic dermatitis during preconception, pregnancy and lactation period. J Eur Acad Dermatol Venereol. 2019 Sep;33(9):1644-59.

5 Australian Product Information - Dupixent ${ }^{\circledR}$ (Dupilumab). June 2020.

6 Koutroulis I, Papoutsis J, Kroumpouzos G. Atopic dermatitis in pregnancy: current status and challenges. Obstet Gynecol Surv. 2011 Oct;66(10):654-63.

7 Babalola 0, Strober BE. Treatment of atopic dermatitis in pregnancy. Dermatol Ther (Heidelb). 2013 JulAug;26(4):293-301.

8 Prabhu S, Suvarna P. Biological agents in pregnancy and lactation - A rational approach. J Skin Sex Transm Dis. 2019;1(2):54-60.

9 Wilder RL. Hormones, pregnancy, and autoimmune diseases. Ann N Y Acad Sci. 1998 May;840(1):45-50.

10 Kage P, Simon JC, Treudler R. A case of atopic eczema treated safely with dupilumab during pregnancy and lactation. J Eur Acad Dermatol Venereol. 2020 Jun;34(6):e256-7.

11 Hamann CR, Egeberg A, Wollenberg A, Gislason G, Skov L, Thyssen JP. Pregnancy complications, treatment characteristics and birth outcomes in women with atopic dermatitis in Denmark. J Eur Acad Dermatol Venereol. 2019 Mar;33(3):577-87.

12 Wollenberg A, Degitz K. [Herpetic eczema in pregnancy]. Dtsch Med Wochenschr. 1995 Oct;120(41):1395-8

13 Ponticelli C, Moroni G. Fetal Toxicity of Immunosuppressive Drugs in Pregnancy. J Clin Med. 2018 Dec;7(12):E552.

14 Koren G, Ornoy A. The role of the placenta in drug transport and fetal drug exposure. Expert Rev Clin Pharmacol. 2018 Apr;11(4):373-85.

15 Porter ML, Lockwood SJ, Kimball AB. Update on biologic safety for patients with psoriasis during pregnancy. Int J Womens Dermatol. 2017 Feb;3(1):21-5.

16 Chambers CD, Johnson DL. Emerging data on the use of anti-tumor necrosis factor-alpha medications in pregnancy. Birth Defects Res A Clin Mol Teratol. 2012 Aug;94(8):607-11.

17 Förger F, Villiger PM. Treatment of rheumatoid arthritis during pregnancy: present and future. Expert Rev Clin Immunol. 2016 Sep;12(9):937-44.

18 Weber-Schoendorfer C, Oppermann M, Wacker E, Bernard N, Beghin D, Cuppers-Maarschalkerweerd B, et al.; network of French pharmacovigilance centres. Pregnancy outcome after TNF- $\alpha$ inhibitor therapy during the first trimester: a prospective multicentre cohort study. Br J Clin Pharmacol. 2015 0ct;80(4):727-39.

19 Nguyen GC, Seow CH, Maxwell C, Huang V, Leung Y, Jones J, et al.; IBD in Pregnancy Consensus Group; Canadian Association of Gastroenterology. The Toronto Consensus Statements for the Management of Inflammatory Bowel Disease in Pregnancy. Gastroenterology. 2016 Mar;150(3):734-757.e1.

20 Blauvelt A, de Bruin-Weller M, Gooderham M, Cather JC, Weisman J, Pariser D, et al. Long-term management of moderate-to-severe atopic dermatitis with dupilumab and concomitant topical corticosteroids (LIBERTY AD CHRONOS): a 1-year, randomised, double-blinded, placebo-controlled, phase 3 trial. Lancet. 2017 Jun;389(10086):2287-303.

21 Simpson EL, Bieber T, Guttman-Yassky E, Beck LA, Blauvelt A, Cork MJ, et al.; SOLO 1 and SOLO 2 Investigators. Two Phase 3 Trials of Dupilumab versus Placebo in Atopic Dermatitis. N Engl J Med. 2016 Dec;375(24):2335-48.

22 Therapeutic Goods Administration [Internet]. Australian categorisation system for prescribing medicines in pregnancy [cited 2020 Oct 5]. Available from: https://www.tga.gov.au/australian-categorisation-systemprescribing-medicines-pregnancy.

23 Therapeutic Goods Administration [Internet]. Australian Public Assessment Report for Dupilumab June 2018 [cited 2020 Oct 5]. Available from: https://www.tga.gov.au/sites/default/files/auspar-dupilumab180612.pdf.

24 Mian M, Dunlap R, Simpson E. Dupilumab for the treatment of severe atopic dermatitis in a pregnant patient: A case report. JAAD Case Rep. 2020 Aug;6(10):1051-2.

25 Middleton PG, Gade EJ, Aguilera C, MacKillop L, Button BM, Coleman C, et al. ERS/TSANZ Task Force Statement on the management of reproduction and pregnancy in women with airways diseases. Eur Respir J. 2020 Feb;55(2):1-24.

\section{Karger'=}




\section{Case Reports in Dermatology}

\begin{tabular}{l|l}
\hline Case Rep Dermatol 2021;13:248-256 \\
\hline DOI: 10.1159/000515246 & $\begin{array}{l}\text { @ 2021 The Author(s). Published by S. Karger AG, Basel } \\
\text { www.karger.com/cde }\end{array}$ \\
\hline
\end{tabular}

Lobo et al:: Atopic Dermatitis Treated Safely with Dupilumab during Pregnancy: A Case Report and Review of the Literature

26 European Medicines Agency (EMA) Committee for Medicinal Products for Human Use. (CHMP) [Internet]. Dupixent Assessment Report July 2017 [cited 2020 0ct 3]. Available from:

https://www.ema.europa.eu/en/documents/assessment-report/dupixent-epar-public-assessmentreport_en.pdf.

27 European Medicines Agency (EMA) Committee for Medicinal Products for Human Use. (CHMP) [Internet]. Dupixent CHMP assessment report on extension of marketing authorisation and an extension of indication variation February 2019 [cited 2020 Oct 3]. Available from:

https://www.ema.europa.eu/en/documents/variationreport/dupixent-h-c-4390-x-0004-g-eparassessment-report-extension_en.pdf.

28 Witzel SJ. Lactation and the use of biologic immunosuppressive medications. Breastfeed Med. 2014 Dec;9(10):543-6.

29 Cheent K, Nolan J, Shariq S, Kiho L, Pal A, Arnold J. Case Report: fatal case of disseminated BCG infection in an infant born to a mother taking infliximab for Crohn's disease. J Crohn's Colitis. 2010 Nov;4(5):603-5.

30 Blauvelt A, Simpson EL, Tyring SK, Purcell LA, Shumel B, Petro CD, et al. Dupilumab does not affect correlates of vaccine-induced immunity: A randomized, placebo-controlled trial in adults with moderate-tosevere atopic dermatitis. J Am Acad Dermatol. 2019 Jan;80(1):158-167.e1.

31 Förger F. Treatment with biologics during pregnancy in patients with rheumatic diseases. Reumatologia. 2017;55(2):57-8.

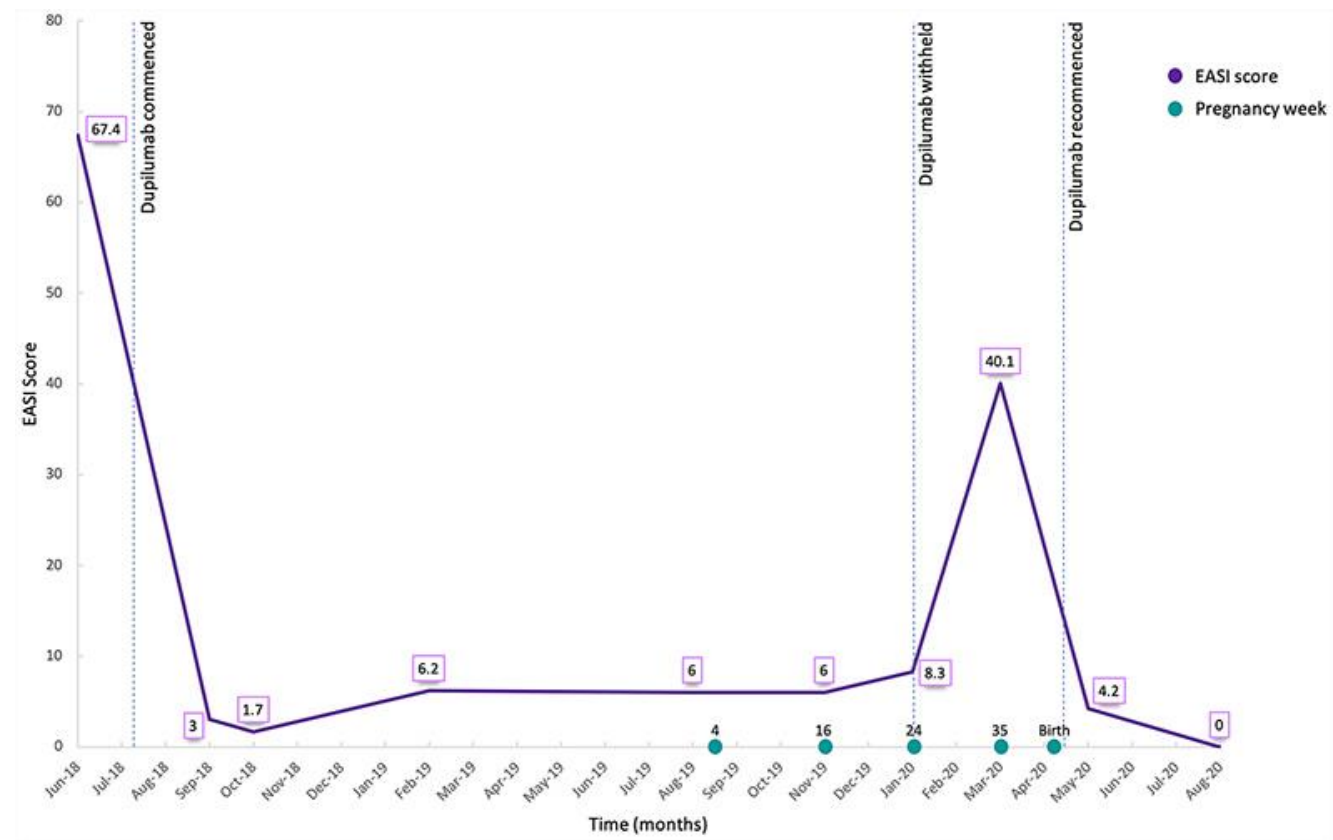

Fig. 1. Timeline showing the influence of treatment with dupilumab on severity of atopic dermatitis before, during, and after pregnancy, as reflected by the Eczema Area and Severity Index (EASI) score. 